\title{
Combined Direct Posterior Split-Gastrocnemius Approach for the Posterolateral Tibial Plateau Involved Fractures
}

This article was published in the following Dove Press journal:

Therapeutics and Clinical Risk Management

\author{
Changhong Chen ${ }^{1,2}$ \\ Lei Huang ${ }^{2}$ \\ Huaqing Zheng ${ }^{2}$ \\ Lin $\mathrm{Liu}^{2}$ \\ Yaofei Chen' \\ Xinhui Xie' \\ Yuntao Wang' \\ 'Department of Orthopedics, Affiliated \\ Zhongda Hospital of Southeast \\ University, Nanjing, Jiangsu 210009 , \\ People's Republic of China; ${ }^{2}$ Department \\ of Orthopedics, Jiangyin Affiliated \\ Hospital of Nanjing University of Chinese \\ Medicine, Wuxi City, Jiangsu Province \\ 214400, People's Republic of China
}

Correspondence: Yuntao Wang; Xinhui

Xie

Email Wangyttod@aliyun.com;

xiexinghuixxh@163.com
Background: Several approaches of fracture reduction and fixation are employed in complex tibial plateau fractures. However, there is a lack of consensus regarding reduction and fixation for fractures to the posterolateral part of the tibial plateau.

Hypothesis: The combined direct posterior split-gastrocnemius approach may be a choice of the posterior part involved comminuted tibial plateau fractures.

Patients and methods: We review cases of 216 patients with tibial plateau fracture and subsequent operation from 2012/1/1 to 2017/1/1. Fifty-six cases involved posterolateral plateau damage. For these 56 patients, we use anteromedial and direct posterior splitgastrocnemius approaches or anterolateral and direct posterior split-gastrocnemius approaches to fix the posterolateral and medial or lateral segments.

Results: From the radiography films, all patients (56/56) achieved a good reduction of the articular surface after surgery (31 patients were anatomic reduction, 25 patients were acceptable reduction). After 12 months follow up, only 2 patients showed poor reduction and no significant difference between $<3$ days after surgery and 12 months follow up. All patients acquired good knee functions at 12 months' time-point of the fracture surgery. These patients have less pain and other related symptoms in daily living according to the Knee Injury and Osteoarthritis Scores.

Conclusion: The direct posterior split-gastrocnemius approach provides efficient and less invasive access to the posterolateral tibial plateau, which is suitable for direct reduction and rigid fixation to the fragments of posterolateral tibial fractures. It is a valuable choice when mapping a surgical approach to tibial plateau fracture reduction involving the posterior tibial plateau and its implementation may offer better post-operative functionality relative to alternative approaches.

Keywords: tibial plateau, posterior split-gastrocnemius approach, posterolateral, outcomes

\section{Introduction}

Tibial plateau fractures involving the posterolateral plateau are not uncommon in adult fractures; computer tomography (CT) scans and magnetic resonance imaging (MRI) studies have demonstrated that the frequency of affecting the posterolateral tibial plateau is from $36 \%$ to $65.2 \%$ in AO/OTA type $\mathrm{B}$ plateau fractures and 54-85.9\% in AO/OTA type C fractures. ${ }^{1,2}$ Although the Schatzker and AO classification schemes are frequently used to describe tibial plateau fractures, they do not provide thorough description of posterolateral fracture fragments (PLFs) and most of the latest researches are focused on posteromedial fracture only. ${ }^{3}$ 3D-CT and 
MRI examinations could help to find many insufficient reductions of posterior fractures, especially in PLFs. ${ }^{4}$ When treating tibia plateau fractures, an anatomic articular reduction and stable fixation are important factors associated with long-term outcome. ${ }^{5}$ A sufficient surgical approach should provide excellent articular visualization and easy fixation for plates, as well as soft tissue preservation and avoidance of complications. Various approaches are used to address tibial plateau fractures including include posteromedial, anterolateral, direct posterior and lateral incisions. ${ }^{6-8}$ However, these approaches may not allow for direct visualization of fracture fragments and a normalizing osteotomy, such as a fibular head osteotomy, is required in these approaches. There is still no consensus or standard guideline when choosing an approach for these PLFs.

In our hospital, we combined direct posterior splitgastrocnemius approach (DPSGA) with other approaches to treat the tibial plateau fractures with posterolateral plateau fragments. ${ }^{7}$ Although this approach involves dissection of the medial neurovascular structures, it can be safely and carefully performed by experienced orthopedists and the most important advantage of this approach is to provide a direct visualization and sufficient fixation space. The objective of this study is to evaluate the reduction and fixation effect of the direct posterior split-gastrocnemius approach combined with other different approaches in complex tibial plateau fractures.

\section{Patients and Methods}

This study is a retrospective study. From January 2012 to January 2017, 216 patients with tibial plateau fracture underwent corrective surgery. Each patient underwent radiographic imaging and $\mathrm{CT}$ scanning at the injury site prior to operation. The classifications were done based on AO/OTA (Figure 1A) and Schatzker (Figure 1B). Tibial plateau fracture with posterolateral fracture fragments is the admission criteria. And the exclusion criteria include: 1. Open tibial plateau fractures. 2. Non-surgical cases. 3. The follow-up time is less than 1 year. We use the axial CT view and find 56 cases of these tibial plateau fractures (25.9\%) involved posterolateral plateau damage. All of these cases were closed fractures without any neurovascular injury or compartment syndrome and followed up at least 1 year after surgery (Table 1 ). The work has been reported in line with the STROCSS criteria. ${ }^{9}$

\section{Surgical Procedure \\ Preoperation and Position}

The patient was placed prone on a well-padded radiolucent table or used the floating position (which was based on a lateral decubitus, and the lower limb was rotated to a prone position when the DPSGA was performed) and a tourniquet was used. ${ }^{10}$ All patients received antibiotic prophylaxis half an hour before the operation.

\section{Fracture Reduction and Fixation}

In the case of medial or lateral tibial plateau fracture with posterolateral plateau fragments, we use double approaches (anteromedial and direct posterior splitgastrocnemius approach or anterolateral and direct posterior split-gastrocnemius approach) to fix the posterolateral and medial or lateral segments. The first step was fracture reduction through an anteromedial or anterolateral tibial approach, after that a T-shaped or reverse L-shaped LCP plate was used to fix the reduced fracture fragments. The next step was the reduction of the PLF. A direct posterolateral incision was used in all of these cases. In this approach, the popliteal neurovascular bundle was protected and the lateral gastrocnemius muscle was retracted medially. The operative interval was between the common peroneal nerve and the lateral gastrocnemius, and the lateral gastrocnemius was retracted after deep dissection. It should be carefully identified the common peroneal nerve and protect it. Then, subperiosteal elevation of the soleus was used to obtain full exposure of the posterolateral fragment. After reducing the PLF, a T-shaped plate was used as a posterior buttress plate and intraoperative fluoroscopic imaging was used to ensure the proper reduction of the fracture and accurate location of the implants. Meanwhile, the varus instability was assessed under fluoroscope.

\section{Postoperative Care}

All patients received thromboprophylaxis in the perioperative period. Patients were immobilized in a knee brace in full extension for 2 weeks. Toe-touching or partial weight bearing was allowed after the first 6 weeks following the operation. Subsequently, full weight bearing was allowed gradually and the patient was referred for physiotherapy.

\section{Measure and Evaluation}

All patients had a good post-operative course without any perioperative complications. Patients' clinical function and radiological film results were assessed at postoperative periods (at $<3$ days and 12 months after surgery). The postoperative 
A

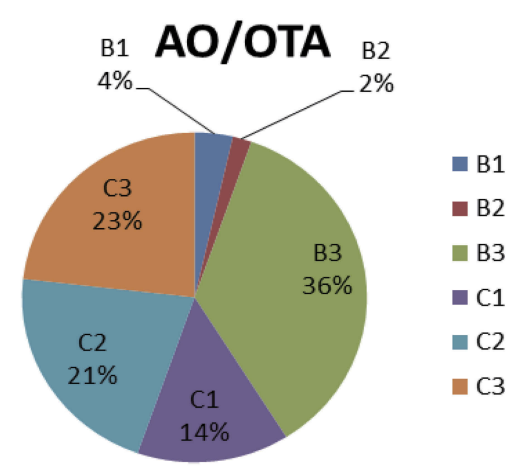

C

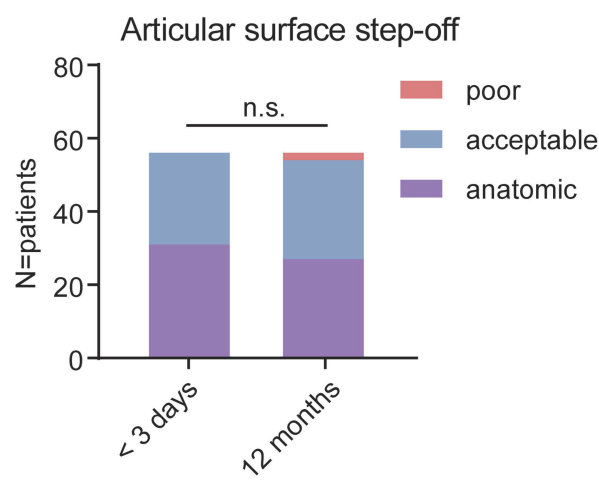

E
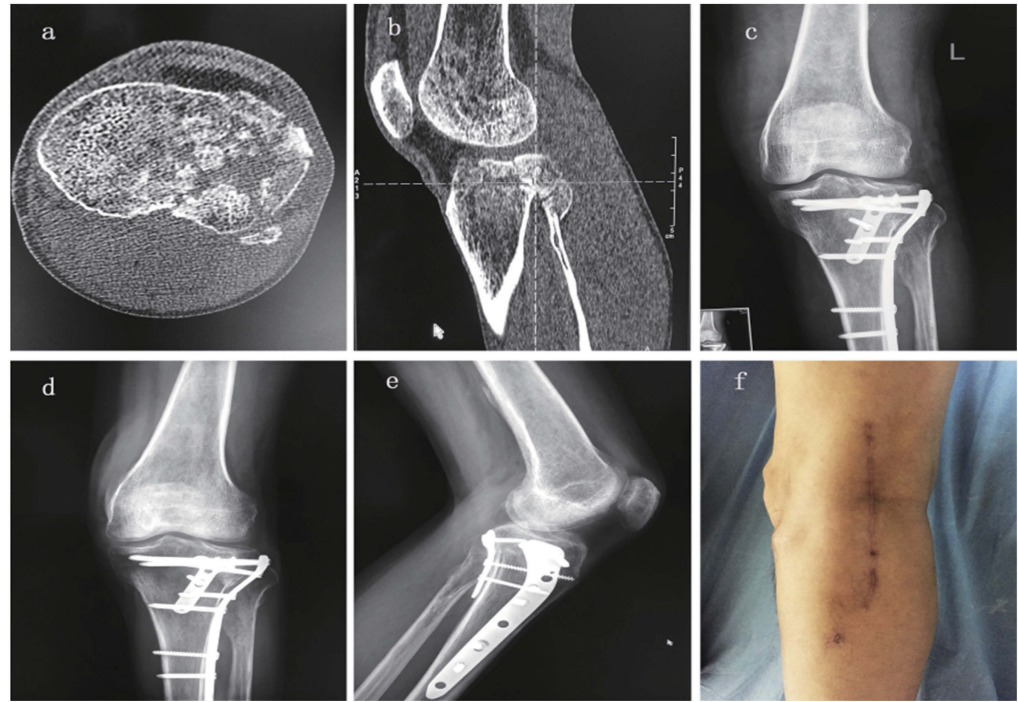

B

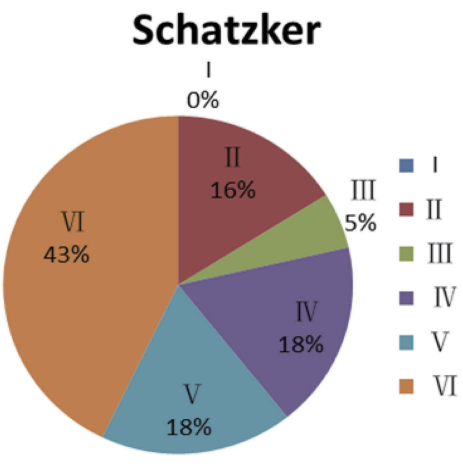

D
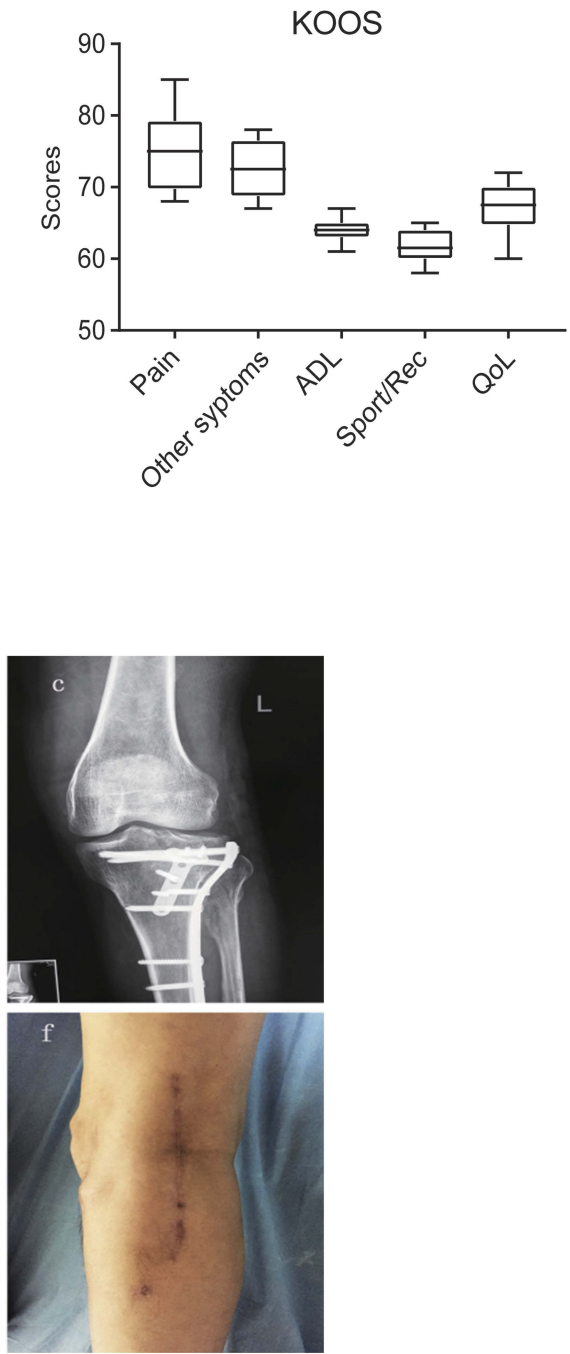

Figure I (A and B) The AO/OTA Classifications and Schatzker Classifications of the patients (cases). (C) Radiological results: The step-offs of joint surfaces on radiograph $(P>0.05)$. (D) The Knee Injury and Osteoarthritis Outcome Score (KOOS) domains. (E) (a and b) A tibial plateau fracture patients with the posterolateral fragment (by CT scan); (c) X-ray film examined 3 days after the operation; $d$ and e. X-ray films examined 12 months after the operation; $f$. The skin scar of the posterolateral incision. Abbreviations: ADL, Function in daily living; QOL, knee-related Quality of life. 
Table I Sample Characteristics

\begin{tabular}{|c|c|c|c|}
\hline & \multirow{2}{*}{$\begin{array}{l}\text { Total } \\
n=56\end{array}$} & \multicolumn{2}{|c|}{ Approaches } \\
\hline & & $\begin{array}{l}\text { AM + } \\
\text { DPSGA }\end{array}$ & $\begin{array}{l}\text { AL + } \\
\text { DPSGA }\end{array}$ \\
\hline Age (years) & $\begin{array}{l}51.2 \text { (range, } \\
16-81 \text { ) }\end{array}$ & 50 & - \\
\hline Male & 29 & 18 & 11 \\
\hline Female & 27 & 15 & 12 \\
\hline Mechanisms of Injury & & & \\
\hline Automobile & 19 & II & 8 \\
\hline Motorcycle & 25 & 14 & 11 \\
\hline Fallen & 8 & 5 & 3 \\
\hline Sport-related & 4 & 3 & I \\
\hline OTA/AO Classification & & & \\
\hline Type BI & 2 & I & I \\
\hline Type B2 & I & I & 0 \\
\hline Type B3 & 20 & 9 & 11 \\
\hline Type CI & 8 & 5 & 3 \\
\hline Type C2 & 12 & 7 & 5 \\
\hline Type C3 & 13 & 10 & 3 \\
\hline Schatzker Classification & & & \\
\hline Type II & 9 & 0 & 9 \\
\hline Type III & 3 & 0 & 3 \\
\hline Type IV & 10 & 10 & 0 \\
\hline Type V & 10 & 7 & 3 \\
\hline Type VI & 24 & 16 & 8 \\
\hline Time to surgery (days) & $\begin{array}{l}7.3 \text { (range, } \\
4-27 \text { ) }\end{array}$ & - & - \\
\hline
\end{tabular}

Abbreviations: AM, anteromedial approach; AL, anterolateral approach; DPSGA, direct posterior split-gastrocnemius approach.

films were evaluated by two independent orthopedists. After the operation, the reduction quality of fracture was evaluated from plain radiograph, according to the step-off of joint surface, which was categorized as anatomic $(0 \mathrm{~mm})$, acceptable $(<2 \mathrm{~mm})$ or poor $(2-4 \mathrm{~mm}){ }^{6}$ At 12 -month follow-up, the Knee Injury and Osteoarthritis Outcome Score (KOOS) questionnaire was used to evaluate the injured knee function. ${ }^{11}$ It consists of 5 subscales: Pain, Other Symptoms, Function in daily living (ADL), Function in sport and recreation (Sport/ Rec) and knee-related Quality of life (QOL).

\section{Statistical Analysis}

SPSS version 20.0 was used for statistical analysis. Descriptive statistics were generated for the demographic and clinical characteristics. Continuous variables were expressed as mean \pm standard deviation and categorical variables were expressed as counts and percentages.
Different time-point data of results were analyzed with two-way ANOVA. A statistically significant difference will be indicated when $\mathrm{P}<0.05$.

\section{Result}

Patients' clinical characteristics were analyzed by homogeneity test and showed no difference between anteromedial approach combined with DPSGA approach and anterolateral approach combined with DPSGA approach (Table 1).

\section{Radiological Results}

The step-off of joint surfaces was measured on radiographs at $<3$ days and 12 months following surgical reduction, there is no significant difference between these two time-point follow-up $(\mathrm{P}>0.05)$ (Figure $1 \mathrm{C}$ and $\mathrm{E})$. Thirtyone patients showed anatomic reduction of the articular surface, 25 patients showed acceptable reduction (1.2 $\pm 0.7 \mathrm{~mm}$ ), no patients showed poor reduction. At the 12 months after the operation, 27 patients still showed anatomic reduction, 27 patients showed acceptable articular surface reduction $(1.7 \pm 0.5 \mathrm{~mm}), 2$ patients showed poor reduction $(3.3 \pm 0.6 \mathrm{~mm})$. No patients accepted second operations.

\section{Clinical Results}

One year after surgery, all patients' knee functions were estimated by use of the Knee Injury and Osteoarthritis Outcome Score (KOOS) questionnaire. The questionnaire consists of 5 subscales: Pain, Other Symptoms, Function in daily living (ADL), Function in sport and recreation (Sport/ $\mathrm{Rec}$ ) and knee-related Quality of life (QOL); each question has graded answers from 0 to 4 and the respondents' scores on the questionnaire can range from 0 to 100 . According to the 1-year follow up, these patients showed good knee functions. The scores of these patients are follows: Pain score (75.14 \pm 5.31$)$, Other Symptoms (72.36 \pm 3.89$)$, ADL (64.01 \pm 1.62$),$ Sport/Rec (61.57 \pm 1.99$)$, QoL (66.86 \pm 3.31$)$ (Figure 1D).

\section{Complications}

Eight patients had grade I post-traumatic osteoarthritis, but no patients have agreed to a second operation. No cases of nerve and/or vessel injury were observed. Surgical site infection did not occur though one patient developed wound-edge skin necrosis that was successfully treated by surgical debridement. 


\section{Discussion}

Among tibial plateau fractures, the posterolateral fracture patterns or fragments are commonly treated through different surgical approaches; however, which approach is optimal for posterolateral fracture patterns is still controversial. ${ }^{12}$ The main goal of the treatment for tibial plateau fractures is to reconstruct the fracture fragments anatomically, restore the extremity axis and achieve strong fixation. ${ }^{5,7}$ The outcomes and long-term successes are commonly affected by the reduction of the fracture segments. An excellent approach must provide direct visualization and enough fixation space. The posterolateral approach, a direct approach without necessitating fibular osteotomy, is a less invasive technique which is suitable for low-energy type fragments of posterolateral tibial fractures. The common peroneal nerve was retracted gently to the lateral side and the lateral gastrocnemius was retracted to the medial side, so that the tension of retraction was mainly on the lateral gastrocnemius muscle and not the common peroneal nerve. This provides an adequate area for the fragments' reduction and internal fixation by the buttress plate. ${ }^{13}$ Previous work in cadavers has demonstrated that, in isolated posterolateral tibial plateau fractures, it is possible to apply anatomical reduction and buttress plating on the posterior surface with a direct posterior split-gastrocnemius approach. Although this approach involves the dissection of the neurovascular bundle, it provides excellent fracture visualization and direct reduction. ${ }^{14}$ With a thorough understanding of the regional anatomy, this approach can be safely performed by experienced orthopedists.

Isolated posterolateral tibial fractures are as severetrauma related injuries easily produce comminuted fractures, so these posterolateral tibial fractures are often seen in conjunction with additional tibial plateau fractures. ${ }^{15}$ The implementation of CT scans used demonstrates that many AO or Shartzker type tibial fractures are combined with posterolateral fracture fragments. ${ }^{16}$ Definitive treatment targets the restoration of the every part of articular surface. A sole incision may not be adequate for the threecolumn fractures (lateral, medial, posterior), especially for posterior fracture fragments. Many case series have advocated the use of combined incision for the treatment of complex tibial plateau fractures involving three parts of plateau. An anterolateral incision plus an anteromedial or posteromedial longitudinal incision have been routinely used for the complex tibial plateau fracture. ${ }^{17,18}$ Medial and lateral incisions better exposed the medial and lateral condyles, especially when the width of plateau is widened, and these approaches allow for easy fracture fragment compression by the plate. However, when the fracture involves the posterior part of the plateau, these approaches cannot optimize the reduction and fixation of posterior column fractures. Particularly in the case of posterolateral coronal fracture, reduction loss appears to occur because reduction and fixation are not easy due to anatomical obstacles, such as the fibula and common peroneal nerve, and the screw cannot rigidly fix the fragments during anterolateral plating. ${ }^{19}$ Numerous authors advocated using a posterior approach to dealing with posterior part fractures. ${ }^{20,21}$

Here, we combined a direct posterior split-gastrocnemius approach for posterolateral fracture fragments in tibial plateau fractures. For this approach, the prone position is posed and a straight incision is made along the border of the medial head of gastrocnemius ending at the level of the joint line. The small saphenous vein is identified in the sulcus between the gastrocnemius heads after incising the popliteal fascia and the medial gastrocnemius is retracted laterally. The semimembranosus complex is retracted medially without detaching its insertion on the posteromedial tibia, and then the posterior lateral tibial plateau is exposed for easy reduction and fixation. This approach provides direct vision and an adequate operative space and satisfactory reduction and rigid fixation for the posterior articular blocks were attainable. Using this approach and combined with anterolateral approach or anteromedial approach, we can cope with any complex type of tibial plateau fracture. Through this kind of surgery, we achieved the anatomical reconstruction of the joint surface, restoration of the extremity axis, easy placement of implants and stable fixation to allow early joint movement, as well as good functional outcomes.

\section{Conclusions}

From this retrospective study, we confirmed that the direct posterior split-gastrocnemius approach, which provides sufficient access to the posterolateral tibial plateau, can provide the sufficient reduction and satisfactory outcome for patients. Although there is currently no consensus regarding the criteria for selecting a surgical approach to address the posterolateral fragment, we put forward this combined direct posterior split-gastrocnemius approach as an optimal choice of approach for comminuted tibial plateau fractures involving the posterior plateau. We do acknowledge the difficulty in evaluating the posterolateral fragments by radiograph and that step-off of articular surface may not completely reflect the joint function. In the future study, we will expand the 
number of cases and use CT scans as one of our evaluation methods at follow-up. And, another shortage of this study is that this is a retrospective cohort study, we think that further randomized controlled studies are needed in the future.

\section{Abbreviations}

CT, computer tomography; MRI, magnetic resonance imaging; PLFs, posterolateral fracture fragments; DPSGA, direct posterior split-gastrocnemius approach; KOOS, Knee Injury and Osteoarthritis Outcome Score; ADL, Function in daily living; QOL, knee-related Quality of life.

\section{Ethics}

All protocols have been approved by the Ethics Committee of Jiangyin Affiliated Hospital of Nanjing University of Chinese Medicine and performed in accordance with ethical standards (NO: 2018065, 2018/06/01). Because of each patient signed a statement before surgery about the allowing of the usage of the medical data, the ethics committee agreed to waive the confirmation of patient written another special informed consent.

\section{Disclosure}

The authors declare no competing interests in this work.

\section{References}

1. Sohn HS, Yoon YC, Cho JW, Cho WT, Oh CW, Oh JK. Incidence and fracture morphology of posterolateral fragments in lateral and bicondylar tibial plateau fractures. J Orthop Trauma. 2015;29(2):91-97. doi:10.1097/BOT.0000000000000170

2. Krause M, Preiss A, Müller G, et al. Intra-articular tibial plateau fracture characteristics according to the "Ten segment classification". Injury. 2016;47(11):2551-2557. doi:10.1016/j.injury.2016.09.014

3. Gicquel T, Najihi N, Vendeuvre T, Teyssedou S, Gayet L-E, Huten D. Tibial plateau fractures: reproducibility of three classifications (Schatzker, AO, Duparc) and a revised Duparc classification. Orthop Trauma Surg Res. 2013;99:805-816. doi:10.1016/j.otsr.2013.06.007

4. Markhardt BK, Gross JM, Monu J. Schatzker classification of tibial plateau fractures: use of CT and MR imaging improves assessment. Radio Graphics. 2009;29(2):585-597. doi:10.1148/rg.292085078

5. Singleton N, Sahakian V, Muir D. Outcome after tibial plateau fracture: how important is restoration of articular congruity? J Orthop Trauma. 2017;31(3):158-163. doi:10.1097/BOT.0000000000000762

6. Qiu W-J, Zhan Y, Sun H, Ya-Feng X, Wang Y-K, Luo C. A posterior reversed L-shaped approach for the tibial plateau fractures-A prospective study of complications (95 cases). Injury Int $J$ Care Inj. 2015;46:1613-1618. doi:10.1016/j
7. Cho J-W, Kim J, Cho W-T, et al. Approaches and fixation of the posterolateral fracture fragment in tibial plateau fractures: a review with an emphasis on rim plating via modified anterolateral approach. Int Orthopaedics. 2017;41:1887-1897. doi:10.1007/s00264-017-3563-6

8. Lin K-C, Tarng Y-W, Lin G-Y, Yang S-W, Hsu C-J, Renn J-H. Prone and direct posterior approach for management of posterior column tibial plateau fractures. Orthopaedics Traumatol. 2015;101:477-482. doi:10.1016/j.otsr.2014.12.021

9. Agha RA, Borrelli MR, Vella-Baldacchino M, Thavayogan R, Orgill DP; for the STROCSS Group. The STROCSS statement: strengthening the reporting of cohort studies in surgery. Int $J$ Surg. 2017;46:198-202. doi:10.1016/j.ijsu.2017.08.586

10. Luo CF, Sun H, Zhang B, Zeng BF. Three-column fixation for complex tibial plateau fractures. $J$ Orthop Trauma. 2010;24:683-692. doi:10.1097/BOT.0b013e3181d436f3

11. Roos EM, Roos PH, Lohmander LS, et al. Knee injury and Osteoarthritis Outcome Score (KOOS): development of a self-administered outcome measure. J Orthop Sports Phys Ther. 1998;28(2):88-96. doi:10.2519/jospt.1998.28.2.88

12. Bishop J, Githens M. Surgical treatment of posterior tibial plateau fractures. Oper Tech Orthop. 2015;25:242-247. doi:10.1053/j. oto.2015.08.004

13. Orapiriyakul W, Apivatthakakul T, Phornphutkul C. Posterolateral tibial plateau fractures, how to buttress? Reversed L posteromedial or the posterolateral approach: a comparative cadaveric study. Arch Orthop Trauma Surg. 2018;138:505-513. doi:10.1007/s00402-0182875-3

14. Özdemir G, Yilmaz B, Sirin E, Keskinöz EN, Kırıkçı G, Bayramoglu A. The anatomical relationship of the neurovascular structures in direct posterior lateral gastrocnemius split approach for posterolateral tibial plateau fractures. Eur J Trauma Emerg Surg. 2018;44(3):427-432. doi:10.1007/s00068-017-0790-x

15. Xiang G, Zhi-Jun P, Qiang Z, et al. Morphological characteristics of posterolateral articular fragments in tibial plateau fractures. Orthopedics. 2013;36(10):e1256-e1261. doi:10.3928/0147744720130920-16

16. Gicquel T, Najihi N, Vendeuvre T, Teyssedou S, Gayet L-E, Huten D. Tibial plateau fractures: reproducibility of three classifications (Schatzker, AO, Duparc) and a revised Duparc classification. Orthopaedics Traumatol. 2013;99(7):805-816. doi:10.1016/j. otsr.2013.06.007

17. Affatato S, Montalti M, Masetti C, Giardina F, Sudanese A. DoubleIncision approach and early rehabilitation in a complicated bicondylar tibial plateau fracture: a case report. Med Princ Pract. 2017;26 (4):387-389. doi:10.1159/000476067

18. Lobenhoffer P, Gerich T, Bertram T, Lattermann C, Pohlemann T, Tscheme H. Particular posteromedial and posterolateral approaches for the treatment of tibial head fractures. Unfallchirurg. 1997;100 (12):957-967. doi:10.1007/s001130050218

19. Kima C-W, Leea C-R, Ana K-C, et al. Predictors of reduction loss in tibial plateau fracture surgery: focusing on posterior coronal fractures. Injury. 2016;47(7):1483-1487. doi:10.1016/j

20. Bhattacharyya T, McCarty LP, Harris MB, et al. The posterior shearing tibial plateau fracture: treatment and results via a posterior approach. J Orthop Trauma. 2005;19(5):305-310.

21. Galla M, Lobenhoffer P. The direct, dorsal approach to the treatment of unstable tibial posteromedial fracture-dislocations. Unfallchirurg. 2003;106(3):241-247. doi:10.1007/s00113-002-0554-9 


\section{Publish your work in this journal}

Therapeutics and Clinical Risk Management is an international, peerreviewed journal of clinical therapeutics and risk management, focusing on concise rapid reporting of clinical studies in all therapeutic areas outcomes, safety, and programs for the effective, safe, and sustained use of medicines. This journal is indexed on PubMed Central, CAS
EMBase, Scopus and the Elsevier Bibliographic databases. The manuscript management system is completely online and includes a very quick and fair peer-review system, which is all easy to use. Visit http://www.dovepress.com/testimonials.php to read real quotes from published authors. 